\title{
AN APPROXIMATION THEOREM FOR INTEGRABLE HARMONIC VECTOR FIELDS
}

\author{
BJÖRN GUSTAFSSON and MAKOTO SAKAI
}

\section{Introduction.}

It is known that every component of a harmonic vector field in a domain $\Omega \subset \mathrm{R}^{N}(N \geqq 2)$ is a harmonic function which has zero net flux across any closed hypersurface in $\Omega$. In the present paper we are concerned with the question whether, conversely, every such harmonic function can be approximated by linear combinations of components of harmonic vector fields.

Our main result concerns approximation in the $L^{1}$-norm (with respect to $N$-dimensional Lebesgue measure) and gives an affirmative answer to the question in case $\Omega$ is bounded, finitely connected and, if $N \geqq 3$, satisfies a mild (but necessary) additional condition. More specifically the result shows that any integrable harmonic function in $\Omega$ with zero flux across any closed hypersurface then can be approximated by linear combinations of the functions (of $x \in \Omega) E_{j}(x-y)$ for $y \in \partial \Omega$ and $1 \leqq j \leqq N$, where $E_{j}=\partial E / \partial x_{j}$ and $E$ denotes the spherically symmetric fundamental solution of (minus) the Laplacian.

This main result is stated in full and proved in $\S 3$. The proof uses a kind of (weighted) converse Poincaré inequality for harmonic functions, which is stated and proved in $\$ 2$. Section one, finally, contains some general observations concerning harmonic vector fields.

\section{Some background.}

By a harmonic vector field in a domain $\Omega \subset \mathrm{R}^{N}(N \geqq 2)$ we mean a vector field $f=\left(f_{1}, \ldots, f_{N}\right): \Omega \rightarrow \mathrm{R}^{N}$ satisfying

$$
\begin{gathered}
\operatorname{div} f=0, \\
\operatorname{rot} f=0
\end{gathered}
$$

(i.e. $\sum_{j=1}^{N} \partial f_{j} / \partial x_{j}=0$ and $\partial f_{j} / \partial x_{k}-\partial f_{k} / \partial x_{j}=0$ for all $k$ and $j$ ). Thus a vector field is a harmonic vector field if and only if it locally is the gradient of a harmonic

Received March 1, 1991. 
function. Each component of a harmonic vector field is a harmonic function, but not every harmonic function is a component of a harmonic vector field (although locally this is true).

We need some notation:

$$
\begin{aligned}
& H(\Omega)=\{\text { harmonic functions in } \Omega\}, \\
& A(\Omega)=\{\text { harmonic vector fields in } \Omega\}, \\
& A(\Omega)_{j}=\left\{f_{j}: f \in A(\Omega)\right\}
\end{aligned}
$$

$(j=1, \ldots, N)$. Thus by the above remarks $A(\Omega)_{j} \subset H(\Omega)$ for all $j$.

When $N=2(1.1)-(1.2)$ reduce to the anti-Cauchy-Riemann equations, so that $f=\left(f_{1}, f_{2}\right) \in A(\Omega)$ if and only if $f_{1}-i f_{2}$ is an analytic function in $\Omega$. Since the operator $\left(f_{1}, f_{2}\right) \mapsto\left(f_{2},-f_{1}\right)$ ("multiplication by $i$ ") acts on $A(\Omega)$ we have $A(\Omega)_{1}=A(\Omega)_{2}$ when $N=2$. Moreover it is well-known that a harmonic function $u$ is the real (or imaginary) part of an analytic function in $\Omega$ if and only if

$$
\int_{\gamma} \frac{\partial u}{\partial n} d s=0
$$

for every closed oriented smooth curve $\gamma$ in $\Omega$.

Thus in two dimensions we have

$$
A(\Omega)_{1}=A(\Omega)_{2}=H(\Omega)_{0},
$$

where $H(\Omega)_{0}$ denotes the set of functions $u \in H(\Omega)$ satisfying (1.3). In higher dimension the situation is slightly more complicated as we shall see.

For arbitrary $N \geqq 2$ set

$$
H(\Omega)_{0}=\left\{u \in H(\Omega): \int_{\Gamma} \frac{\partial u}{\partial n} d \sigma=0\right. \text { for }
$$

$$
\text { every closed oriented smooth hypersurface } \Gamma \text { in } \Omega \text { \}, }
$$

where $\partial u / \partial n$ denotes the normal derivative on $\Gamma$ in the direction singled out by the orientation and where $d \sigma$ denotes the hypersurface measure on $\Gamma$.

Using differential forms and the Hodge's star operator [Wa] the condition in (1.5) can also be written $\int_{r} * d u=0$ for every closed oriented smooth hypersurface $\Gamma$ in $\Omega$. By de Rham's theorem [Wa] this condition is equivalent to the (closed) $(N-1)$-form $* d u$ being exact, i.e. to the existence of an $(N-2)$-form $v$ satisfying

$$
* d u=d v
$$


When $N=2 v$ is the usual harmonic conjugate function of $u$ (determined up to an additive constant) and (1.6) is the Cauchy-Riemann system. For $N \geqq 3 v$ can be regarded as some kind of generalized harmonic conjugate of $u$, but is far from uniquely determined.

It was observed in [Gu 1, p. 238] that $A(\Omega)_{j} \subset H(\Omega)_{0}$ for all $j$. For convenience let us reproduce the simple proof. Let $f \in A(\Omega)$ and consider $f_{1}$ for example. With $\Gamma$ as above and using (1.1), (1.2) and Stokes' theorem we get

$$
\begin{aligned}
\int_{\Gamma} \frac{\partial f_{1}}{\partial n} d \sigma= & \int_{\Gamma} \sum_{j=1}^{N}(-1)^{j-1} \frac{\partial f_{1}}{\partial x_{j}} d x_{1} \ldots \widehat{d x_{j}} \ldots d x_{N} \\
= & \int_{\Gamma} \frac{\partial f_{1}}{\partial x_{1}} d x_{2} \ldots d x_{N}+\sum_{j=2}^{N}(-1)^{j-1} \int_{\Gamma} \frac{\partial f_{j}}{\partial x_{1}} d x_{1} \ldots \widehat{d x_{j}} \ldots d x_{N} \\
= & \int_{\Gamma} \frac{\partial f_{1}}{\partial x_{1}} d x_{2} \ldots d x_{N}+\sum_{j=2}^{N}(-1)^{j-1} \int_{\Gamma} d\left(f_{j} d x_{2} \ldots \widehat{d x_{j}} \ldots d x_{N}\right) \\
& -\sum_{j=2}^{N}(-1)^{j-1} \int_{\Gamma}(-1)^{j} \frac{\partial f_{j}}{\partial x_{j}} d x_{2} \ldots d x_{N}=0,
\end{aligned}
$$

as desired $\left(d x_{1} \ldots \widehat{d x}_{j} \ldots d x_{N}\right.$ means $\left.d x_{1} \ldots d x_{j-1} d x_{j+1} \ldots d x_{N}\right)$.

Another observation (made in [Gu2]) is that when $N \geqq 3$ then $A(\Omega)_{j}$ in general depends on $j$ (even if $\Omega$ is rotationally symmetric). Consider e.g. a punctured ball, say $\Omega=B(0, r) \backslash\{0\}$. When $N \geqq 3 \Omega$ is simply connected, hence every $f \in A(\Omega)$ can be written $f=\nabla u$ for some $u \in H(\Omega)$. Taking into account what kind of singularities at $x=0$ which are possible for $u$ one easily sees that among those functions in $\Omega$ which do not behave worse than $|x|^{1-N}$ at $x=0$ we have in $A(\Omega)_{j}$, for any fixed $1 \leqq j \leqq N$, only functions of the form

$$
a_{j} \frac{x_{j}}{|x|^{N}}+u_{j}
$$

with $a_{j} \in \mathrm{R}$ and $u_{j} \in H(B(0, r))$. From this it is clear that $A(\Omega)_{k} \neq A(\Omega)_{j}$ when $k \neq j$ in the present case.

Thus in higher dimension we should not consider the individual $A(\Omega)_{j}$ but rather their sum $A(\Omega)_{1}+\ldots+A(\Omega)_{N}$. Since $A(\Omega)_{j} \subset H(\Omega)_{0}$ for each $j$ we have

$$
A(\Omega)_{1}+\ldots+A(\Omega)_{N} \subset H(\Omega)_{0} .
$$

We do not know whether the inclusion in (1.7) can be strict. However we have the following. 
Proposition. Let $\Omega \subset \mathrm{R}^{N}$ be an arbitrary domain. Then $A(\Omega)_{1}+\ldots+A(\Omega)_{N}$ is dense in $H(\Omega)_{0}$ in the topology of uniform convergence on compact sets.

ProOF. Let

$$
E(x)=\left\{\begin{array}{lll}
c_{2} \log \frac{1}{|x|} & \text { if } \quad N=2 \\
\frac{c_{N}}{|x|^{N-2}} & \text { if } \quad N \geqq 3
\end{array}\right.
$$

so that $-\Delta E=\delta_{0}$, the Dirac measure at the origin (hence $c_{N}>0$ ). Also, set

$$
E_{j}=\frac{\partial E}{\partial x_{j}}
$$

so that $\nabla E=\left(E_{1}, \ldots, E_{N}\right)$. Then, as functions of $x, E(x-y) \in H(\Omega), \nabla E(x-y) \epsilon$ $A(\Omega), E_{j}(x-y) \in A(\Omega)_{j}$ for $y \in \Omega^{c}$. Since equations (1.1), (1.2) (and the Laplace equation) have constant coefficients any derivative of a function in any of the above spaces remain in the same space.

It follows that for any $y \in \Omega^{c}$ and any multiindex $\alpha$ with $|\alpha| \geqq 1$

$$
D^{\alpha} E(x-y) \in A(\Omega)_{1}+\ldots+A(\Omega)_{N}
$$

as a function of $x$.

Now we shall actually prove that the linear span of the functions in (1.8) is dense in $H(\Omega)_{0}$. Let $L: H(\Omega)_{0} \rightarrow \mathrm{R}$ be a continuous linear functional which annihilates all the functions in (1.8). We then have to prove that $L=0$.

$L$ can be represented by a (signed) measure $\mu$ with supp $\mu \subset \Omega$ so that $L(u)=\int u d \mu$ for $u \in H(\Omega)_{0}$. Thus by (1.8)

$$
\int D^{\alpha} E(x-y) d \mu(x)=0
$$

for every $y \in \Omega^{c}$ and $|\alpha| \geqq 1$. In terms of the Newtonian potential $U^{\mu}=E * \mu$ of $\mu$ this simply says that

$$
D^{\alpha} U^{\mu}=0 \text { on } \Omega^{c}
$$

for every $|\alpha| \geqq 1$.

Let $N$ be an open set with smooth boundary $\partial N$ and satisfying supp $\mu \subset N \subset \subset \Omega$ and such that each component of $N^{c}$ meets $\Omega^{c}$. Obseve that $U^{\mu}$ is harmonic and hence real analytic in a neighbourhood of $N^{c}$. Therefore (1.9) implies that $U^{\mu}$ is constant in each component of $N^{c}$. In particular $\partial U^{\mu} / \partial n=0$ on $\partial N$ and $U^{\mu}$ is constant on each component of $\partial N$. For any $u \in H(\Omega)_{0}$ we therefore obtain 
$L(u)=\int u d \mu=-\int_{N} u \cdot \Delta U^{\mu} d m=-\int_{N} U^{\mu} \cdot \Delta u d m-\int_{\partial N} u \frac{\partial U^{\mu}}{\partial n} d \sigma+\int_{\partial N} U^{\mu} \frac{\partial u}{\partial n} d \sigma=0$,

as desired ( $m$ denotes $N$-dimensional Lebesgue measure).

REMARK. Observe that we actually proved that the linear span of the functions in (1.8) is dense in $H(\Omega)_{0}$. Even more, one may e.g. choose just one point $y$ in each component of $\Omega^{c}$ and use only the $D^{\alpha} E(x-y)$ then obtained (for all $|\alpha| \geqq 1$ ). Also note that all the functions in (1.8) are components of vector fields in $A(\Omega)$ which globally (in $\Omega$ ) are gradients of harmonic functions. Thus the proposition remains true with $A(\Omega)$ replaced by the set of gradients of harmonic functions (i.e., with $A(\Omega)_{j}$ replaced by $\left.\left\{\frac{\partial u}{\partial x_{j}}: u \in H(\Omega)\right\}\right)$.

\section{An estimate.}

Set

$$
\begin{gathered}
H L^{p}(\Omega)=H(\Omega) \cap L^{p}(\Omega) \\
H L^{p}(\Omega)_{0}=H(\Omega)_{0} \cap L^{p}(\Omega) \\
A L^{p}(\Omega)=A(\Omega) \cap L^{p}(\Omega)^{N} \\
A L^{p}(\Omega)_{j}=\left\{f_{j}: f \in A L^{p}(\Omega)\right\}
\end{gathered}
$$

for $1 \leqq p \leqq \infty$ and where $L^{p}(\Omega)=L^{p}(\Omega ; m)$. Clearly $A L^{p}(\Omega)_{j} \subset A(\Omega)_{j} \cap L^{p}(\Omega)$, and in general strict inclusion holds because, taking $N=2$ and $\Omega$ simply connected for example, a harmonic function may be in $L^{p}(\Omega)$ without its harmonic conjugate being there.

By (1.7)

$$
A L^{p}(\Omega)_{1}+\ldots+A L^{p}(\Omega)_{N} \subset H L^{p}(\Omega)_{0}
$$

and in [ $\mathrm{Gu} 2]$ the question was raised whether the left member in (2.1) is dense in the right member when $p=1$ and $\Omega$ is bounded. Our main result states that this is the case under certain assumptions on $\Omega$. For the proof we need the following lemma which might be of independent interest.

LEMMA. Let $\Omega \subset \mathrm{R}^{N}$ be any bounded open set and set

$$
\delta(x)=\operatorname{dist}\left(x, \Omega^{c}\right)
$$

Then there exists a constant $C$ such that 


$$
\int_{\Omega} \delta(x)|\nabla u(x)| d m(x) \leqq C \int_{\Omega}|u| d m
$$

for all $u \in H L^{1}(\Omega)$.

REMARK. The above estimate easily generalizes to

$$
\|\delta \nabla u\|_{L^{p}} \leqq C\|u\|_{L^{p}}
$$

for any $1 \leqq p \leqq \infty\left(\right.$ and all $\left.u \in H L^{p}(\Omega)\right)$.

ProOF. Set

$$
\begin{aligned}
& \Omega_{0}=\{x \in \Omega: \delta(x)>1\}, \\
& \Omega_{n}=\left\{x \in \Omega: 2^{-n}<\delta(x) \leqq 2^{-n+1}\right\}
\end{aligned}
$$

$(n=1,2, \ldots)$. Let $\alpha$ be a fixed large number. (Some of the assertions below depend on $\alpha$ being sufficiently large.) For each $n$ let $\left\{Q_{n 1}, Q_{n 2}, \ldots, Q_{n j_{n}}\right\}$ be those cubes of the type $Q_{k_{1} \ldots k_{N}}=\left\{x \in \mathrm{R}^{N}: \frac{k_{i}}{\alpha 2^{n}} \leqq x_{i} \leqq \frac{k_{i}+1}{\alpha 2^{n}}(1 \leqq i \leqq N)\right\}\left(k_{i} \in \mathrm{Z}\right)$ which meet $\Omega_{n}$. Then

$$
\Omega_{n} \subset \bigcup_{j=1}^{j_{n}} Q_{n j} \subset \Omega
$$

$(n=0,1, \ldots)$. Let $c_{n j}$ denote the center of $Q_{n j}$.

Let $u \in H L^{1}(\Omega)$. Then

$$
\int_{\Omega} \delta(x)|\nabla u(x)| d m(x) \leqq \sum_{n=0}^{\infty} \sum_{j=1}^{j_{n}} \int_{Q_{n j}} \delta(x)|\nabla u(x)| d m(x)
$$

and we shall estimate the integrals to the right. First of all we have

$$
\delta(x) \leqq 2^{-n+2} \text { for } x \in Q_{n j} \text {. }
$$

As to $\nabla u(x)$ it follows from the Poisson integral formula that there exists a constant $C$ (independent of $x$ and $r$ ) such that

$$
|\nabla u(x)| \leqq \frac{C}{r} \sup _{B(x ; r)}|u|
$$

whenever $B(x ; r) \subset \Omega$. ((2.5), (2.6) by the way immediately give (2.3) in the case $p=\infty$.) For each $y \in B(x ; r)$ the meanvalue property gives that 


$$
|u(y)| \leqq \frac{C}{r^{N}} \int_{B(y ; r)}|u| d m
$$

for some other constant $C$ and assuming that $B(x ; 2 r) \subset \Omega$. Throughout the proof the letter $C$ will denote various constants which are different in different formulas. Combining (2.6) and (2.7) gives

$$
|\nabla u(x)| \leqq \frac{C}{r^{N+1}} \int_{B(x ; 2 r)}|u| d m,
$$

holding whenever $B(x ; 2 r) \subset \Omega$.

Next we can find a number $\beta>1$ such that $B\left(x ; \frac{1}{\alpha 2^{n}}\right) \subset B\left(c_{n j} ; \frac{\beta}{\alpha 2^{n}}\right)$ whenever $x \in Q_{n j}$. Choosing then $2 r=\frac{1}{\alpha 2^{n}}$ in (2.8) and setting $B_{n j}=B\left(c_{n j} ; \frac{\beta}{\alpha 2^{n}}\right)$ gives

$$
|\nabla u(x)| \leqq C\left(\alpha 2^{n}\right)^{N+1} \int_{B_{n j}}|u| d m
$$

for $x \in Q_{n j}$. Observe that $B_{n j} \subset \Omega$ if $\alpha$ was chosen large enough. $\beta$ can be chosen independently of $\alpha$; in fact any $\beta \geqq \frac{\sqrt{N}}{2}+1$ works.

Combining (2.5) and (2.9) and integrating over $Q_{n j}$ gives

$$
\int_{Q_{n j}} \delta(x)|\nabla u(x)| d m(x) \leqq C \cdot \alpha \int_{B_{n j}}|u| d m .
$$

Now taking the union over all $j$ and $n$

$$
\bigcup_{n, j} B_{n j}=\Omega
$$

and it is easy to see that there is an upper bound for the overlappings between the $B_{n j}$ :

$$
\sum_{n, j} \chi_{B_{n j}} \leqq C<\infty .
$$

By this the desired inequality (2.2) follows directly from (2.10). 


\section{The main result.}

Throughout this section $\Omega$ will be assumed to be a bounded domain in $\mathrm{R}^{N}(N \geqq 2)$ such that $\Omega^{c}$ has only finitely many components. Let $S$ denote the linear span of the functions (of $x \in \Omega$ ) $E_{j}(x-y)$ for $y \in \partial \Omega$ and $1 \leqq j \leqq N$ and let $\widetilde{S}$ denote the linear span of $S$ together with all functions of the form $E(x-y)-E(x-z)$ with $y, z \in \partial \Omega$ belonging to the same component of $\Omega^{c}$. Then

$$
\begin{aligned}
& S \subset A L^{1}(\Omega)_{1}+\ldots+A L^{1}(\Omega)_{N} \subset H L^{1}(\Omega)_{0}, \\
& S \subset \tilde{S} \subset H L^{1}(\Omega)_{0} .
\end{aligned}
$$

It was proved in [Gu 2, Lemma 1.3] that $S$ is dense in $A L^{1}(\Omega)_{1}+\ldots+A L^{1}(\Omega)_{N}$. Thus $A L(\Omega)_{1}+\ldots+A L^{1}(\Omega)_{N}$ is dense in $H L^{1}(\Omega)_{0}$ if and only if $S$ is dense in $H L^{1}(\Omega)_{0}$. Our main result below shows that this is the case under mild topological assumptions on $\Omega$ but not in complete generality (if $N \geqq 3$ ).

THEOREM. With $\Omega, S$ and $\tilde{S}$ as above

(i) $\tilde{S}$ is dense in $H L^{1}(\Omega)_{0}$.

(ii) If $N=2$ then even $S$ is dense in $H L^{1}(\Omega)_{0}$.

(iii) If $N \geqq 3$ then $S$ is dense in $H L^{1}(\Omega)_{0}$ provided $\Omega$ satisfies some suitable additional assumption, e.g. the following.

For each component $K$ of $\Omega^{c}$ there is a dense subset $K^{*}$ of $K$ such that any two points in $K^{*}$ can be joined by a rectifiable arc within $K$.

(iv) For every $N \geqq 3$ there are examples of domains $\Omega$ (not satisfying (3.1)) for which $S$ is not dense in $H L^{1}(\Omega)_{0}$.

Note that (3.1) holds e.g. if $\Omega$ equals the interior of its closure. A necessary and sufficient, but less explicit, condition for $S$ to be dense in $\tilde{S}$ is given by (3.12). The proof below is based on a technique which has earlier been used, for related results, in [Be], [He], [Sa 1-3], [Gu 2].

Proof. Any continuous linear functional on $H L^{1}(\Omega)_{0}$ can be represented by a function $g \in L^{\infty}(\Omega)$. That $g$ annihilates $S$ means that $\int_{\Omega} E_{j}(x-y) g(x) d m(x)=0$ for all $y \in \partial \Omega$ and all $1 \leqq j \leqq N$ and that it annihilates $\tilde{S}$ means that moreover $\int_{\Omega}(E(x-y)-E(x-z)) g(x) d m(x)=0$ whenever $y, z \in \partial \Omega$ belong to the same component of $\Omega^{c}$. Denoting the components of $\Omega^{c}$ by $K_{1}, \ldots, K_{r}$ and extending $g$ by zero outside $\Omega$ we thus see that, in terms of the Newtonian potential $U^{g}$ of $g$, $g$ annihilates $S$ if and only if

$$
\nabla U^{g}=0 \text { on } \partial \Omega,
$$

and $g$ annihilates $\tilde{S}$ if and only if (3.2) and

$$
U^{g}=c_{j} \quad \text { on } \quad \partial K_{j} \quad(1 \leqq j \leqq r)
$$


hold for some constants $c_{1}, \ldots, c_{r}$. Observe that $\partial K_{j}=(\partial \Omega) \cap K_{j}$.

Since $g \in L^{\infty}(\Omega) U^{g}$ and $\nabla U^{g}$ are continuous everywhere and harmonic in the interior of $\Omega^{c}$. Moreover $\left|\nabla U^{g}(x)\right| \rightarrow 0$ as $|x| \rightarrow+\infty$. Therefore the maximum principle shows that (3.2) implies that

$$
\nabla U^{g}=0 \text { on } \Omega^{c} \text {. }
$$

(Hence (3.2) and (3.4) are equivalent.) Note that (3.4) implies that $U^{g}=$ constant $(=0)$ in a neighbourhood of infinity. Therefore it also follows from the maximum principle that (3.2) and (3.3) together imply

$$
U^{g}=c_{j} \quad \text { on } \quad K_{j} \quad(1 \leqq j \leqq r) .
$$

Thus it follows that $g \in L^{\infty}(\Omega)$ annihilates $S$ if and only if (3.4) holds and annihilates $\tilde{S}$ if and only if (3.4) and (3.5) hold (for some $c_{j}$ ).

Now, to prove (i) of the theorem it is enough, by the Hahn-Banach theorem, to prove that $g \in L^{\infty}(\Omega)$ annihilates all $H L^{1}(\Omega)_{0}$ whenever it annihilates $\tilde{S}$, i.e. to show that (3.4) and (3.5) imply that

$$
\int_{\Omega} u g d m=0 \text { for all } u \in H L^{1}(\Omega)_{0} .
$$

So assume that (3.4) and (3.5) hold. We first need some estimates.

It is well-known [Gü] (and elementary to prove) that

$$
\left|\nabla U^{g}(x)-\nabla U^{g}(y)\right| \leqq C|x-y| \log \frac{1}{|x-y|}
$$

for $|x-y|<e^{-2}$. To avoid some inconveniences when dist $(x, \Omega)$ is not small we now redefine $\delta(x)$ to be the minimum of dist $(x, \Omega)$ and $e^{-2}$. Then (3.4), (3.7) give

$$
\left|\nabla U^{g}(x)\right| \leqq C \delta(x) \log \frac{1}{\delta(x)} \quad(x \in \Omega) .
$$

Moreover, combining (3.8) with (3.5) gives

$$
\left|U^{g}(x)-c_{j}\right| \leqq C \delta(x)^{2} \log \frac{1}{\delta(x)}
$$

when $x \in \Omega$ is close to $K_{j}$.

In order to integrate by parts in $\int_{\Omega} u g d m=-\int u \Delta U^{g} d m$ we need certain cutoff functions $\omega_{n}$ with decay matching (3.8), (3.9). (The construction of these goes back to Ahlfors and Bers [Be]; see also [He]). Choose a function $\psi \in C^{\infty}(\mathrm{R})$ satisfying $0 \leqq \psi \leqq 1, \psi(t)=0$ for $t \leqq 1, \psi(t)=1$ for $t \geqq 2$ and set 


$$
\omega_{n}(x)=\psi\left(\frac{n}{\log \log \frac{1}{\delta(x)}}\right)
$$

for $x \in \Omega$. Then $\omega_{n}$ is Lipschitz continuous, has compact support in $\Omega$ and $\lim \omega_{n}(x)=1$ for each $x \in \Omega$. A straightforward computation shows that $\lim _{n \rightarrow \infty}$

$$
\left|\nabla \omega_{n}(x)\right| \leqq \frac{C}{n \delta(x) \log \frac{1}{\delta(x)}} \quad(x \in \Omega) .
$$

Now consider the left member of (3.6). Using dominated convergence, the definition of $U^{g}$ and integration by parts we get

$$
\begin{aligned}
\int_{\Omega} u g d m & =\lim _{n \rightarrow \infty} \int_{\Omega} \omega_{n} u g d m \\
& =-\lim _{n \rightarrow \infty} \int_{\Omega} \omega_{n} u \Delta U^{g} d m \\
& =\lim _{n \rightarrow \infty}\left[\int_{\Omega} u \nabla \omega_{n} \nabla U^{g} d m+\int_{\Omega} \omega_{n} \nabla u \nabla U^{g} d m\right] \\
& =\lim _{n \rightarrow \infty}\left[\int_{\Omega} u \nabla \omega_{n} \nabla U^{g} d m-\right. \\
& \left.-\int_{\Omega} U^{g} \nabla \omega_{n} \nabla u d m-\int_{\Omega} \omega_{n} U^{g} \Delta u d m\right] \\
& =\lim _{n \rightarrow \infty}\left[\int_{\Omega} u \nabla \omega_{n} \nabla U^{g} d m-\int_{\Omega} U^{g} \nabla \omega_{n} \nabla u d m\right] .
\end{aligned}
$$

Here the first term vanishes (in the limit) as a consequence of (3.8), (3.10) and the integrability of $u$.

As to the second term, choose small (disjoint) neighbourhoods $N_{j}$ of the $K_{j}(1 \leqq j \leqq r)$ in $\Omega$. Observe that $\operatorname{supp} \nabla \omega_{n} \subset \bigcup_{j=1}^{r} N_{j}$ and $\omega_{n}=1$ in $\Omega \backslash \bigcup_{j=1}^{r} N_{j}$ for $n$ large enough. Thus we get 


$$
\begin{aligned}
& \left|\int_{\Omega} U^{g} \nabla \omega_{n} \nabla u d m\right| \leqq \sum_{j=1}^{r}\left|c_{j} \int_{N_{j}} \nabla \omega_{n} \nabla u d m\right|+ \\
& +\sum_{j=1}^{r}\left|\int_{N_{j}}\left(U^{g}-c_{j}\right) \nabla \omega_{n} \nabla u d m\right|
\end{aligned}
$$

where

$$
\begin{aligned}
\int_{N_{j}} \nabla \omega_{n} \nabla u d m & =\int_{\partial N_{j}} \omega_{n} \frac{\partial u}{\partial n} d \sigma-\int_{N_{j}} \omega_{n} \Delta u d m \\
& =\int_{\left(\partial N_{j}\right) \cap \Omega} \frac{\partial u}{\partial n} d \sigma=0
\end{aligned}
$$

for large $n$, and

$$
\begin{aligned}
& \left|\int_{N_{j}}\left(U^{g}-c_{j}\right) \nabla \omega_{n} \nabla u d m\right| \leqq \\
& \leqq \frac{C}{n} \int_{N_{j}} \delta(x)|\nabla u| d m \leqq \frac{C}{n} \int_{\Omega}|u| d m \leqq \\
& \leqq \frac{C}{n} \rightarrow 0
\end{aligned}
$$

by (3.9), (3.10) and the lemma in $\S 2$. Thus also the second term in (3.11) vanishes (in the limit) and (3.6) is proved. This proves (i) of the theorem.

To prove (ii) it is, by (i), enough to prove that $S$ is dense in $\tilde{S}$ when $N=2$. So let $y$ and $z$ be two points on $\partial \Omega$ belonging to the same component, say $K$, of $\Omega^{c}$. Then we have to show that $\log |x-y|-\log |x-z|$ can be approximated by linear combinations of functions in $S$. For any small $\varepsilon>0$ choose $y^{\prime \prime} \in B(y ; \varepsilon) \cap \Omega$ and $z^{\prime \prime} \in B(z ; \varepsilon) \cap \Omega$. Let $y^{\prime}, z^{\prime}$ be points on $K$ which minimize the distance to $y^{\prime \prime}$ and $z^{\prime \prime}$ respectively. Then $\left|y^{\prime}-y\right|<2 \varepsilon,\left|z^{\prime}-z\right|<2 \varepsilon$ and the (open) line segments $y^{\prime} y^{\prime \prime}$ and $z^{\prime} z^{\prime \prime}$ are contained in $K^{c}$. Since $\varepsilon$ is arbitrary is enough if we can approximate $u(x)=\log \left|x-y^{\prime}\right|-\log \left|x-z^{\prime}\right|$ by functions in $S$.

Clearly $y^{\prime \prime}$ and $z^{\prime \prime}$ can be joined by, say, a polygonal line within $K^{c}$. By the preceeding paragraph this line can be extended to a polygonal line $\gamma$ joining $y^{\prime}$ and $z^{\prime}$. Except for the endpoints $\left(y^{\prime}\right.$ and $\left.z^{\prime}\right) \gamma$ lies entirely in $K^{c}$. 
Since $K$ is connected $u$ has a single-valued branch $v(x)$ in $K^{c}$ of its harmonic conjugate $\arg \left(x-y^{\prime}\right)-\arg \left(x-z^{\prime}\right)$. The existence of the polygonal line $\gamma$ easily implies that $v$ is bounded in $K^{c}$. In fact, $v$ is certainly bounded on $\gamma$, say $|v| \leqq M<\infty$ on $\gamma$, and we claim that $|v(x)|$ never exceeds $M+4 \pi$ anywhere in $K^{c}$.

To prove this, let $x_{0}$ be an arbitrary point in $K^{c}$. We may assume that $x_{0} \in(K \cup \gamma)^{c}$. Let $w(x)$ be a single-valued branch of $\arg \left(x-y^{\prime}\right)-\arg \left(x-z^{\prime}\right)$ in $\gamma^{c}$ such that $w(x)=v(x)$ in a neighbourhood of $x_{0}$. Now $K^{c}$ is connected and $\gamma$ separates $K^{c}$, so $(K \cup \gamma)^{c}$ has exactly two components. In the component to which $x_{0}$ belongs we have $v(x)=w(x)$ and in the other one $v(x)=w(x)+2 \pi$ or $v(x)=w(x)-2 \pi$ (since we can reach that component from the first one by crossing $\gamma$ ). We may further assume that $x_{0}$ is not on the straight line passing through $y^{\prime}$ and $z^{\prime}$. The straight line $L$ passing through $x_{0}$ and $\left(y^{\prime}+z^{\prime}\right) / 2$ must intersect $\gamma$. Let $y_{0}$ be a first point of intersection, counted from $x_{0}$, so that the open segment $x_{0} y_{0}$ does not meet $\gamma$, and let $w\left(y_{0}\right)$ denote the boundary value of $w$ at $y_{0}$ along this segment. Then $\left|w\left(y_{0}\right)-w\left(x_{0}\right)\right| \leqq 2 \pi$ and hence $\left|v\left(x_{0}\right)\right|=\left|w\left(x_{0}\right)\right| \leqq$ $\left|w\left(y_{0}\right)\right|+2 \pi \leqq\left|v\left(y_{0}\right)\right|+4 \pi \leqq M+4 \pi$, as claimed.

The above shows that $u+i v$ is an integrable analytic function in $\Omega((u,-v) \epsilon$ $A L^{1}(\Omega)$ in our notation). Therefore by a theorem of Bers [Be] $u+i v$ can be approximated by complex linear combinations of $1 /(x-\xi)$ with $\xi \in \partial \Omega$ (here $x$ and $\xi$ are regarded as complex numbers). Taking the real part of this approximation we obtain the desired approximation of $u$. This completes the proof of (ii).

To prove (iii) first notice, by the Hahn-Banach theorem and by what was said in the beginning of the proof, that $S$ is dense in $\tilde{S}$ if and only if (3.4) implies (3.5) for any $g \in L^{\infty}(\Omega)$. This implication is a condition on $\Omega$, which can be stated in a form free from $g$ as follows.

\section{If a function $u \in C^{1}\left(\mathrm{R}^{N}\right)$ satisfies $\Delta u \in L^{\infty}\left(\mathrm{R}^{N}\right)$ and $\nabla u=0$ on $\Omega^{c}$ then $u$ is constant on each component of $\Omega^{c}$.}

In fact, if (3.12) holds then taking $u=U^{g}\left(g \in L^{\infty}(\Omega)\right)$ shows that (3.4) implies (3.5). Conversely, assume that (3.4) implies (3.5), let $u \in C^{1}\left(R^{N}\right), \Delta u \in L^{\infty}\left(R^{N}\right)$, $\nabla u=0$ on $\Omega^{c}$ and we shall see that $u$ is constant on each component of $\Omega^{c}$. Clearly $u$ is constant in a neighbourhood of infinity and we may assume that this constant is zero. Since $\Delta u \in L^{\infty}\left(R^{N}\right), u$ is in the Sobolev space $W_{\text {loc }}^{2, p}\left(R^{N}\right)$ for every $p<\infty$, and by [Ki-St, Lemma A.4 p. 53] the assumption $\nabla u=0$ on $\Omega^{c}$ then implies that $\Delta u=\operatorname{div} \nabla u=0$ a.e. on $\Omega^{c}$. Setting $g=-\Delta u$ it follows that $g \in L^{\infty}\left(R^{N}\right), u=U^{g}$ and that $g=0$ a.e. on $\Omega^{c}$. Since $\nabla U^{g}=\nabla u=0$ on $\Omega^{c}, U^{g}$ satisfies (3.4), hence it satisfies (3.5), hence $u$ is constant on each component of $\Omega^{c}$ as desired.

Thus, $S$ is dense in $\tilde{S}$ (and hence in $\left.H L^{1}(\Omega)_{0}\right)$ if and only if (3.12) holds.

To finish the proof of (iii) one just has to observe that (3.1) implies (3.12). In fact, if $u \in C^{1}\left(\mathrm{R}^{N}\right)$ and $\nabla u=0$ on $K$ then by (3.1) $\nabla u$ can be integrated between any two 
points in $K^{*}$ yielding that $u$ is constant on $K^{*}$, and then, by continuity, it is constant also on $K$.

As to (iv) Whitney [Wh] gives an example of a function $u \in C^{N-1}\left(R^{N}\right)$ such that $\nabla u=0$ on a compact non-rectifiable arc $K$ but nevertheless $u$ is not constant on $K$. This $u$ can be taken to vanish identically outside a compact set. If $N \geqq 3$ then $u \in C^{2}\left(R^{N}\right)$ so that $\Delta u \in L^{\infty}\left(R^{N}\right)$. Thus we get a counterexample to (3.12), with e.g. $\Omega=B(0 ; r) \backslash K$ for $r$ large enough. Hence $S$ is not always dense in $\tilde{S}$ when $N \geqq 3$.

This completes the proof of the theorem.

RemarK. Note that we have proved that (3.12) always holds when $N=2$. Thus, in Whitney's example [Wh], $\Delta u$ is not in $L^{\infty}\left(\mathrm{R}^{2}\right)$ when $N=2$.

ACKNOWLeDGMent. We are grateful to Göran Gustafssons stiftelse and the Bar-Ilan University (Ramat-Gan, Israel) for generous visiting appointments of importance for the present work and to The Swedish Natural Research Council for continuous support. We also want to thank Siv Sandvik for typing our manuscript.

\section{REFERENCES}

[Be] L. Bers, An approximation theorem, J. Analyse Math. 14 (1965), 1-4.

[Gu1] B. Gustafsson, An ill-posed moving boundary problem for doubly-connected domains, Ark. Mat. 25 (1987), 231-253.

[Gu2] B. Gustafsson, On quadrature domains and an inverse problem in potential theory, J. Analyse Math. 55 (1990), 172-216.

[Gü] N. M. Günter, Potential Theory and its Applications to Basic Problems of Mathematical Physics, Frederick Ungar Publ. Co., New York, 1967.

[He] L. I. Hedberg, Approximation in the mean by solutions of elliptic equations, Duke Math. J. 40 (1973), 9-16.

[Ki-St] D. Kinderlehrer and G. Stampacchia, An Introduction to Variational Inequalities and their Applications, Academic Press, New York, 1980.

[Sa1] M. Sakai, Quadrature Domains, Lecture Notes in Math, vol. 934, Springer-Verlag, Berlin, 1982.

[Sa2] M. Sakai, Applications of variational inequalities to the existence theorem on quadrature domains, Trans. Amer. Math. Soc. 276 (1983), 267-279.

[Sa3] M. Sakai, Solutions to the obstacle problem as Green potentials, J. Analyse Math. 44(1984/85), 97-116.

[Wa] F. W. Warner, Foundations of Differentiable Manifolds and Lie Groups, Scott, Foresman and Company, Glenview, 1971.

[Wh] H. Whitney, A function not constant on a connected set of critical points, Duke Math. J 1 (1935), 514-517.

MATEMATISKA INSTITUTIONEN KUNGL. TEKNISKA HOGSKOLAN S-10044 STOCKHOLM SWEDEN
DEPARTMENT OF MATHEMATICS TOKOY METROPOLITAN UNIVERSITY MINAMIOSAWA, HACHIOJI-SHI TOKOY, 192-03 